\title{
Topological terms and diffeomorphism anomalies in fluid dynamics and sigma models
}

\author{
V.P. Nair $\odot^{*}$ \\ Physics Department, City College of the CUNY, New York, New York 10031, USA
}

(Received 9 September 2020; accepted 23 February 2021; published 26 April 2021)

\begin{abstract}
The requirement of diffeomorphism symmetry for the target space can lead to anomalous commutators for the energy-momentum tensor for sigma models and for fluid dynamics, if certain topological terms are added to the action. We analyze several examples. A particular topological term is shown to lead to the known effective hydrodynamics of a dense collection of vortices, i.e., the vortex fluid theory in $2+1$ dimensions. The possibility of a similar vortex fluid in $3+1$ dimensions, as well as a fluid of knots and links, with possible extended diffeomorphism algebras is also discussed.
\end{abstract}

DOI: 10.1103/PhysRevD.103.085017

\section{INTRODUCTION}

The components of the energy-momentum tensor in fluid dynamics or in a field theory will obey commutation rules which express the fact that they are the generators of diffeomorphisms. Anomalies in diffeomorphism symmetries will be reflected, in a Hamiltonian formulation of the theory, as anomalous commutators. Although we generally seek to avoid such anomalies for reasons of unitarity, the following more nuanced situation can arise. The fields or fluid variables we are considering are maps from spacetime, denoted as $M$, into a target manifold $\mathcal{M}$. As is well known in the context of sigma models, the choice of local coordinates on $\mathcal{M}$ should not affect physical results, such as the $S$-matrix. In other words, field redefinitions via diffeomorphisms of $\mathcal{M}$ are possible. It is then possible that there are certain types of topological terms which can be included in the action and which can create an incompatibility between diffeomorphisms in spacetime $M$ and on the target space $\mathcal{M}$. This feature can then be manifest as anomalous commutation rules for the energymomentum tensor. Such topological terms are the subject of this paper.

The immediate motivation comes from the work of Wiegmann, and Wiegmann and Abanov, who considered vortices in a superfluid, and for the quantum Hall system, in $2+1$ dimensions [1]. In a situation with a large number of vortices, it is possible to consider an effective hydrodynamics for them. In other words, each vortex can be

\footnotetext{
*vpnair@ccny.cuny.edu
}

Published by the American Physical Society under the terms of the Creative Commons Attribution 4.0 International license. Further distribution of this work must maintain attribution to the author(s) and the published article's title, journal citation, and DOI. Funded by SCOAP . viewed as a point particle and a fluid with such constituents is obtained. This fluid is different from the underlying fluid which produced the vortices in the first place. The authors of [1] showed that the commutation rules for the energymomentum tensor for the vortex fluid has anomalous terms. We may recall that anomalous commutators can be viewed as 2-cocycle terms obtained via the descent equations from an index density in two higher dimensions, and hence, they are closely tied to the existence of gravitational anomalies [2]. Since there are no purely gravitational anomalies in $2+1$ dimensions [3], how is it possible to have anomalous commutators? Could they arise from the incompatibility mentioned above?

There is also a larger context for our analysis in view of the recent resurgence of interest in fluid dynamics. Studies of the behavior of a quantum Hall droplet as an incompressible fluid with the possibility of nondissipative viscosity in $2+1$ dimensions [4] and the holographic fluid-gravity correspondence in the AdS/CFT framework [5] have been two major tracks for ongoing research. Added to this is the fact that a formalism for non-Abelian fluid dynamics incorporating anomalous symmetries [6] is clearly the natural framework for interesting physical phenomena such as the chiral magnetic effect and its variants [7]. And, of course, the fluid version of the Wess-Zumino term as an effective action for anomalies is the classic example of a topological term which can influence the dynamics of a fluid $[8,9]$. In the present analysis, we will focus on a slightly different class of topological terms. We will consider terms which can lead to anomalous commutators as well as terms which can couple different fluids. Notice that the example of the vortex fluid may be considered as a two-fluid system with the fundamental underlying fluid and the vortex fluid, so it should be interesting to analyze systems with independent dynamics for each component except for coupling via topological terms. 
A useful observation is that anomalous commutators define 2-cocycles in the operator algebra [3]. For the equaltime algebra for a theory in $3+1$ dimensions, we should thus consider a five-form which is closed but not exact. Locally such a five-form can be written as the exterior derivative of a four-form $\Gamma$. This can be added to the action, and can lead to anomalous commutators. Thus, in $3+1$ dimensions, our strategy will be to consider sigma models or fluid variables for which we can identify nontrivial five-forms. For $2+1$ dimensions, we will need nontrivial four-forms.

This paper is organized as follows. In Sec. II, we analyze the sigma model with target space $\mathbb{C P}^{2}$, showing how the extended version of the diffeomorphism algebra arises and how it is connected to diffeomorphism of the target space. In Sec. III, we consider various types of topological terms which can be added to the standard action for fluid dynamics. This is done in terms of a group-theoretic formulation of the Clebsch variables, which helps to simplify the analysis. Section IV is devoted to the case of one of the topological terms and the corresponding extended version of the diffeomorphism algebra is obtained. In Sec. V., we carry out the necessary comparison to identify this case with the vortex fluid work of [1] in $2+1$ dimensions, and also show that a special case yields a central extension identified in [10] for $3+1$ dimensions. In Sec. VI, we analyze the other topological term, designated $I_{2}$, and argue that the extended algebra obtained may apply for an effective hydrodynamics of knots and links in $3+1$ dimensions. The paper concludes with a short discussion.

\section{A SIGMA MODEL ON $\mathbb{C P}^{2}$}

We will start with a sigma model in $2+1$ dimensions with the target space $\mathcal{M}$ as the complex projective space $\mathbb{C P}^{2}$. This will serve as a concrete example which sets the paradigm for later discussion. The space $\mathbb{C P}^{2}$ has nontrivial $H^{4}$ and a generating element of this can be taken as $\Omega \wedge \Omega$, where $\Omega$ is the Kähler two-form. We can think of $\mathbb{C P}^{2}$ as $S U(3) / U(2)$ and use a group element $U \in S U(3)$ with the identification $U \sim U h, h \in U(2) \subset S U(3)$ to coordinatize the manifold. In a $3 \times 3$ matrix representation of $U$, the Kähler one-form is given by

$$
A=i \frac{2}{\sqrt{3}} \operatorname{Tr}\left(t_{8} U^{-1} d U\right), \quad t_{8}=\frac{1}{2 \sqrt{3}}\left[\begin{array}{ccc}
1 & 0 & 0 \\
0 & 1 & 0 \\
0 & 0 & -2
\end{array}\right] .
$$

Under $U \rightarrow U h, A$ is not invariant, but transforms as

$$
A(U h)=A(U)-\frac{1}{\sqrt{3}} d \theta_{8}
$$

where $h=\exp \left(i t_{8} \theta_{8}+i t_{i} \theta_{i}\right), i=1,2,3$. Thus $A$ is not a one-form on the coset $S U(3) / U(2)$, but Kähler two-form
$\Omega=d A$ is invariant under $U \rightarrow U h$ and is well defined on $\mathbb{C P}^{2}$. One can introduce local coordinates for the manifold by writing

$$
U_{i 3}=\frac{1}{\sqrt{1+\bar{z} \cdot z}}\left(z^{1}, z^{2}, 1\right) .
$$

It is also useful to consider real coordinates defined by, say, $z^{1}=\varphi^{1}+i \varphi^{2}, z^{2}=\varphi^{3}+i \varphi^{4}$. In terms of these parametrizations, the one-form $A$ can be written as

$$
\begin{aligned}
A & =-\frac{i}{2} \frac{\bar{z} \cdot d z-z \cdot d \bar{z}}{(1+\bar{z} \cdot z)}=-J_{a b} \frac{\varphi^{a} d \varphi^{b}}{\left(1+\varphi^{2}\right)}, \\
J_{12} & =-J_{21}=1, \quad J_{34}=-J_{43}=1, \quad \text { all other } J_{a b}=0 .
\end{aligned}
$$

The product $\Omega \wedge \Omega$ can be written as $d \Gamma$ where $\Gamma=A \wedge d A$. While $\Omega \wedge \Omega$ is well defined on $\mathbb{C P}^{2}, \Gamma$ does not descend to the coset space since $A$ is not invariant under $U \rightarrow U h . \Gamma$ will be the topological term we add to the action. Thus the theory we are considering is defined by the action

$$
S=\frac{1}{2} \int d^{3} x G_{a b} \partial_{\mu} \varphi^{a} \partial^{\mu} \varphi^{b}+k \int \Gamma
$$

where $k$ is a constant and $G_{a b}$ is the metric tensor for the target space $\mathbb{C P}^{2}$. Notice that $\Gamma$ shifts by a total derivative under $U \rightarrow U h$, so that the bulk action is well defined with appropriate boundary conditions. We take the fields to vanish at spatial infinity. The surface terms on equal-time spatial slices do not necessarily vanish and can lead to a canonical transformation. In terms of the real coordinates $\varphi^{a}, \Gamma$ is given by

$$
\begin{aligned}
\Gamma & =\frac{1}{3} \epsilon_{a b c d} \frac{\varphi^{a} d \varphi^{b} d \varphi^{c} d \varphi^{d}}{\left(1+\varphi^{2}\right)^{2}} \\
& =\frac{1}{3} \epsilon_{a b c d} \frac{\varphi^{a} \partial_{\mu} \varphi^{b} \partial_{\nu} \varphi^{c} \partial_{\alpha} \varphi^{d}}{\left(1+\varphi^{2}\right)^{2}} d x^{\mu} \wedge d x^{\nu} \wedge d x^{\alpha} .
\end{aligned}
$$

The canonical momentum $\Pi_{a}$ can be read off from the action as

$$
\begin{gathered}
d^{2} x \Pi_{a}=d^{2} x G_{a b} \dot{\varphi}^{a}-\Gamma_{a}, \\
\Gamma_{a}=k \epsilon_{a b c d} \frac{\varphi^{b} d \varphi^{c} d \varphi^{d}}{\left(1+\varphi^{2}\right)^{2}} \\
=k \epsilon_{a b c d} \frac{\varphi^{b} \partial_{i} \varphi^{c} \partial_{j} \varphi^{d}}{\left(1+\varphi^{2}\right)^{2}} d x^{i} \wedge d x^{j} .
\end{gathered}
$$

(The differentials $d x$ in $\Gamma_{a}$ in this equation are for the spatial coordinates only.)

The term $\Gamma$ we have added is a differential form on spacetime and is therefore independent of the spacetime metric. Therefore it will not contribute to the energymomentum tensor. By considering the variation of the 
action with respect to the spacetime metric, we obtain the energy-momentum tensor as

$$
T_{\mu \nu}=G_{a b} \partial_{\mu} \varphi^{a} \partial_{\nu} \varphi^{b}-\eta_{\mu \nu} \frac{1}{2}(G \partial \varphi \partial \varphi) .
$$

The momentum density which can be identified as the generator of spatial diffeomorphisms is given by $T_{i 0}=G_{a b} \partial_{i} \varphi^{a} \dot{\varphi}^{b}$. The generator of the transformation $x^{i} \rightarrow x^{i}+\xi^{i}$ is thus given by

$$
\begin{aligned}
T(\xi) & =\int\left(\xi^{i} \partial_{i} \varphi^{a}\right) G_{a b} \dot{\varphi}^{b}=\int\left(\xi^{i} \partial_{i} \varphi^{a}\right)\left(\Pi_{a}+\Gamma_{a}\right) \\
& =\int\left(\xi \cdot \partial \varphi^{a}\right)\left(-i \frac{\delta}{\delta \varphi^{a}}+\Gamma_{a}\right)=-i \int\left(\xi \cdot \partial \varphi^{a}\right) D_{a} \\
D_{a} & =\left(\frac{\delta}{\delta \varphi^{a}}+i \Gamma_{a}\right) .
\end{aligned}
$$

$D_{a}$ is a covariant derivative for the target space with $\Gamma_{a}$ as the gauge field.

It is now completely straightforward to calculate the commutator of two such generators. We find

$\left[T(\xi), T\left(\xi^{\prime}\right)\right]=i T\left(\left[\xi, \xi^{\prime}\right]\right)-\int \rho^{a}(x) \sigma^{b}(y)\left[D_{a}, D_{b}\right]$

where $\left[\xi, \xi^{\prime}\right]^{i}=\xi \cdot \partial \xi^{\prime i}-\xi^{\prime} \cdot \partial \xi^{i}, \quad \rho^{a}=\left(\xi \cdot \partial \varphi^{a}\right)$, and $\sigma^{b}=\left(\xi^{\prime} \cdot \partial \varphi^{b}\right)$. We can think of $\Gamma_{a}$ as a connection or gauge field on the space of fields and hence the commutator $\left[D_{a}, D_{b}\right]$ is the field strength,

$$
\left[D_{a}, D_{b}\right]=i\left(\frac{\partial \Gamma_{b}(y)}{\partial \varphi^{a}(x)}-\frac{\partial \Gamma_{a}(x)}{\partial \varphi^{b}(y)}\right) \equiv i \mathcal{F}_{a b}(x, y) .
$$

It is simpler to use the notation of differential forms for the target space and write the connection as

$$
\mathcal{A}=\int \Gamma_{a} \delta \varphi^{a}
$$

where $\delta$ denotes the exterior derivative for the space of fields. What we need for the curvature (12) is thus $\delta \mathcal{A}$. In terms of $\Omega=\frac{1}{2} \Omega_{a b} d \varphi^{a} d \varphi^{b}$, we can write $\mathcal{A}$ as

$$
\mathcal{A}=k \int\left[A_{a} \delta \varphi^{a} \Omega+\Omega_{k l} \delta \varphi^{k} d \varphi^{l} A\right] .
$$

[We do not write the wedge sign any more to avoid too much clutter; it is taken as understood. Notice that the comparison of (13) and (14) gives another expression for $\Gamma_{a}$ as well.] To obtain the curvature, we may note the following identities:

$$
\begin{aligned}
\delta\left(A_{a} \delta \varphi^{a}\right) & =\frac{1}{2} \Omega_{a b} \delta \varphi^{a} \delta \varphi^{b}, \\
\delta \Omega & =d\left(\Omega_{k l} \delta \varphi^{k} d \varphi^{l}\right), \\
\delta\left(\Omega_{k l} \delta \varphi^{k} d \varphi^{l}\right) & =-d\left(\frac{1}{2} \Omega_{k l} \delta \varphi^{k} \delta \varphi^{l}\right), \\
\delta\left(A_{a} d \varphi^{a}\right) & =d\left(A_{a} \delta \varphi^{a}\right)+\Omega_{a b} \delta \varphi^{a} d \varphi^{b} .
\end{aligned}
$$

Using these results we can calculate $\delta \mathcal{A}$ as

$$
\begin{aligned}
\delta \mathcal{A}= & k \int\left[\frac{1}{2} \Omega_{a b} \delta \varphi^{a} \delta \varphi^{b} \Omega_{k l} d \varphi^{k} d \varphi^{l}\right. \\
& \left.-\Omega_{a b} \delta \varphi^{a} d \varphi^{b} \Omega_{k l} \delta \varphi^{k} d \varphi^{l}\right] .
\end{aligned}
$$

Some total derivatives in the integrand have been dropped since they integrate to zero. We assume the boundary conditions are such that this is the case. The second term on the right-hand side of (11) can now be written as

$$
\begin{aligned}
& -\int \rho^{a}(x) \sigma^{b}(y)\left[D_{a}, D_{b}\right] \\
& =-i k \int\left[\frac{1}{2} \Omega_{a b} \rho^{a} \sigma^{b} \Omega_{k l} d \varphi^{k} d \varphi^{l}-2 \Omega_{a b} \rho^{a} d \varphi^{b} \Omega_{k l} \sigma^{k} d \varphi^{l}\right] \\
& \left.\left.=-i k V_{\rho}\right\rfloor V_{\sigma}\right\rfloor \mathcal{F}
\end{aligned}
$$

where the symbol $\left.V_{\rho}\right\rfloor$ denotes the interior contraction with the functional vector field

$$
V_{\rho}=\int \rho^{a} \frac{\delta}{\delta \varphi^{a}}
$$

Explicitly, for a (functional) differential form $F=$ $\int \frac{1}{2} F_{a b} \delta \varphi^{a} \delta \varphi^{b}$

$$
\begin{aligned}
\left.V_{\rho}\right\rfloor F & \left.=\int \rho^{a} \frac{\delta}{\delta \varphi^{a}}\right\rfloor \int \frac{1}{2} F_{a b} \delta \varphi^{a} \delta \varphi^{b} \\
& =\frac{1}{2} \int\left[F_{a b} \rho^{a} \delta \varphi^{b}-F_{a b} \delta \varphi^{a} \rho^{b}\right] \\
& =\int F_{a b} \rho^{a} \delta \varphi^{b} .
\end{aligned}
$$

Consider now the differential four-form $\Omega^{2}$ on the target space. We use contractions with $V_{\rho}$ and $V_{\sigma}$ and write it as a differential form on space by taking $\varphi^{a}$ as functions of the coordinates; i.e., we pull back the result to spatial manifold. We can then easily check that

$$
\left.\left.\left.\left.V_{\rho}\right\rfloor V_{\sigma}\right\rfloor \mathcal{F}=V_{\rho}\right\rfloor V_{\sigma}\right\rfloor(\Omega \Omega) .
$$

We can now rewrite (11) for the commutator of the generators of spatial diffeomorphisms as 


$$
\left.\left.\left[T(\xi), T\left(\xi^{\prime}\right)\right]=i T\left(\left[\xi, \xi^{\prime}\right]\right)-i k \int V_{\rho}\right\rfloor V_{\sigma}\right\rfloor\left(\Omega^{2}\right) .
$$

The first term on the right-hand side is what is expected from the fact that a diffeomorphism $x^{i} \rightarrow x^{i}+\xi^{i}$ on a space-dependent function $f$ leads to the change

$$
\delta f=\xi^{i} \frac{\partial}{\partial x^{i}} f(x) .
$$

The second term on the right-hand side of (21) shows that the generators $T(\xi)$ for the diffeomorphisms have anomalous commutation rules, the anomaly being related to the $H^{4}$ element of the target space $\mathcal{M}$. The definition of the generator as in (10) also shows how this anomaly can be avoided. Define

$\mathcal{T}(\xi)=T(\xi)-\int\left(\xi \cdot \partial \varphi^{a}\right) \Gamma_{a}=-i \int\left(\xi \cdot \partial \varphi^{a}\right) \frac{\delta}{\delta \varphi^{a}}$.

It is then trivial to see that $\left[\mathcal{T}(\xi), \mathcal{T}\left(\xi^{\prime}\right)\right]=i \mathcal{T}\left(\left[\xi, \xi^{\prime}\right]\right)$, with no anomalous terms. However, $\mathcal{T}$ is related to the components of the energy-momentum tensor via the subtraction of the integral of $\left(\xi \cdot \partial \varphi^{a}\right) \Gamma_{a}$. Since $\Gamma_{a}$ is not well defined on $\mathbb{C P}^{2}$, as we have mentioned after (2), this redefinition is problematic. In other words, $\mathcal{A}$ is a gauge field on the space of field configurations and hence not invariant under field redefinitions or target space diffeomorphisms. Thus, while the use of $\mathcal{T}(\xi)$ will eliminate the anomaly for diffeomorphisms of the spatial manifold, we lose the freedom of field redefinitions or target space diffeomorphisms.

This is the key result of this section. We can add to the action a term $\int \mathcal{A}_{a} \dot{\varphi}^{a}$ where $\mathcal{A}$ is the potential for an element of $H^{4}$ (or $H^{d+1}$ for $d$-dimensional spacetime) of the target space. This can lead to a conflict between diffeomorphisms of the base spatial manifold and the space of field configurations, resulting in anomalous commutators. In the next two sections, we will explore a similar structure for fluids in $2+1$ and $3+1$ dimensions.

\section{THE NATURE OF POSSIBLE TOPOLOGICAL TERMS FOR FLUIDS}

We start with fluids in $3+1$ dimensions; the $2+1$ dimensional case can be easily obtained by a simple reduction.

In the classic Lagrange approach to fluid dynamics, one considers a multiparticle system, where $x^{i}(z, t)$ denotes the position of the $z$ th particle at time $t$, where $z$ is an element of some indexing set labeling the particles. When the number of particles is very large and a continuum approximation is meaningful, one chooses the initial positions of the particles as the label for the particle. In other words, $x^{i}(z, 0)=z^{i}$. Thus $x^{i}(z, t)$ may be regarded as the image of $z^{i}$ under a diffeomorphism parametrized by the time coordinate $t$. The kinetic term in the action takes the usual form

$$
S_{\text {kin }}=\frac{1}{2} \int d^{3} z \rho_{0}(z) \dot{x}_{i} \dot{x}^{i}
$$

We take the particle mass to be 1 and $\rho_{0}(z)$ gives the number density of particles as a function of the fiducial variables $z^{i}$. The canonical one-form at the level of particles is obviously given by

$$
\mathcal{A}=\int d^{3} z \rho_{0}(z) v_{i} \delta x^{i}=\int d^{3} z \rho_{0}(z) \dot{x}_{i} \delta x^{i}
$$

The use of the notation $\delta x^{i}$ rather than $d x^{i}$ signifies that this is to be viewed as a one-form on the space of configurations. If the helicity of the fluid system is fixed, then the velocity admits the Clebsch parametrization

$$
v_{i}=\partial_{i} \theta+\alpha \partial_{i} \beta
$$

for arbitrary functions $\theta, \alpha, \beta$. The canonical one-form, for this parametrization, reduces to

$$
\begin{aligned}
\mathcal{A} & =\int d^{3} z \rho_{0}(z)\left(\partial_{i} \theta+\alpha \partial_{i} \beta\right) \delta x^{i}=\int d^{3} z \rho_{0}(z)(\delta \theta+\alpha \delta \beta) \\
& =\int d^{3} x \rho(x)(\delta \theta+\alpha \delta \beta)
\end{aligned}
$$

where the density $\rho(x)$, as a function of the $x$ coordinates, is defined by $d^{3} z \rho_{0}(z)=d^{3} x \rho(x)$. This shows that to obtain the canonical one-form as in (27) we should take the term in the action involving time derivatives to be $\int \rho \dot{\theta}+\rho \alpha \dot{\beta}$. A suitable action for fluid dynamics (in terms of the Eulerian variables) is then

$$
S[\rho, \theta, \alpha, \beta]=\int \rho \dot{\theta}+\rho \alpha \dot{\beta}-\left[\frac{1}{2} \rho v^{2}-V\right] .
$$

Here we have also included a term corresponding to the potential energy. This expression gives the action suitable for the Clebsch parametrization with $(\rho, \theta),(\rho \alpha, \beta)$ forming two sets of canonically conjugate variables. For more on actions for fluids and the derivation of the standard fluid equations of motion, including the emergence of the convective derivative, from (28), see [6,11].

There is a group-theoretic version of the Clebsch parametrization which is also useful. Towards this, consider the group $S U(1,1)$. A typical element $g$ may be parametrized as

$$
g=\frac{1}{\sqrt{1-\bar{u} u}}\left[\begin{array}{ll}
1 & u \\
\bar{u} & 1
\end{array}\right]\left[\begin{array}{cc}
e^{i \theta / 2} & 0 \\
0 & e^{-i \theta / 2}
\end{array}\right]
$$

where $u$ is a complex variable. It is easy to verify that 


$$
\begin{aligned}
& -i \operatorname{Tr}\left(\sigma_{3} g^{-1} d g\right)=d \theta+\alpha d \beta, \\
& \alpha=\frac{\bar{u} u}{1-\bar{u} u}, \quad \beta=(-i / 2) \ln \left(\frac{u}{\bar{u}}\right) .
\end{aligned}
$$

The variable $\theta$ corresponds to the compact direction, or $U(1)$ subgroup generated by the Pauli matrix $\sigma_{3} ; \alpha$ and $\beta$ parametrize $S U(1,1) / U(1)$. The action (28) can be written in terms of $g$ as

$S\left[J^{\mu}, g\right]=-i \int J^{\mu} \operatorname{Tr}\left(\sigma_{3} g^{-1} \partial_{\mu} g\right)-\int\left[\frac{J_{i} J_{i}}{2 \rho}+V\right]$

where we denote $J^{0}=\rho . J_{i}$ can be eliminated by its equation of motion and leads back to the form in (28). [The action (31) is to be viewed as a functional of the currents $J^{\mu}$ and the group element $g$. Upon elimination of $J_{i}$, it becomes a functional of $J^{0}=\rho$ and $g$ (i.e., $\alpha, \beta, \theta$ ) as in (28).] It is also easy to make a relativistic generalization, with the action given by

$$
S=-i \int J^{\mu} \operatorname{Tr}\left(\sigma_{3} g^{-1} \partial_{\mu} g\right)-F(n)
$$

where $F(n)$ is a function of the variable $n$, which is defined by $J^{\mu} J_{\mu}=n^{2}$. The function $F(n)$ will characterize the fluid. ${ }^{1}$ We will not discuss this in any more detail, except to note that the $T_{i 0}$ component of the energy-momentum tensor for (32) is given by

$$
T_{i 0}=\rho\left(\partial_{i} \theta+\alpha \partial_{i} \beta\right) .
$$

Given that $(\rho, \theta),(\rho \alpha, \beta)$ are canonical pairs, we verify easily that

$$
\left[T(\xi), T\left(\xi^{\prime}\right)\right]=i T\left(\left[\xi, \xi^{\prime}\right]\right), \quad T(\xi)=\int \xi^{i} T_{i 0} .
$$

Our aim is to consider topological terms which one can add to the action (31), or (32), and which can potentially lead to anomalous commutation rules for diffeomorphisms. However, a comment is in order, before we move on. The compact $U(1)$ direction of the $S U(1,1)$ may be a bit puzzling, since the classical Clebsch parametrization does not have a compactness requirement. Using (27), we get

$$
[\rho(f), g(x)]=-i g(x) \frac{\sigma_{3}}{2} f(x), \quad \rho(f)=\int f(x) \rho(x) .
$$

This means that in the quantum theory

$$
\mathcal{U}^{\dagger} g \mathcal{U}=g e^{i \pi \sigma_{3}}=-g
$$

\footnotetext{
${ }^{1}$ For a general discussion about using group-theoretic variables for fluid dynamics, see $[6,8]$.
}

with $\mathcal{U}=\exp \left[-2 \pi i \int \rho\right]$. All observables involve even powers of $g$, and so are invariant under the action of $\mathcal{U}$. Effectively, we can set $\mathcal{U}=1$, giving $\int \rho=N$ for some integer $N$. This is equivalent to saying that the fluid is made of particles with $\rho$ being the number density. Since this is what happens in reality, we regard the existence of a compact direction as a good feature, justifying the use of $S U(1,1)$. [If the total vorticity is also quantized we should use $S U(2)$ in place of $S U(1,1)$.]

Turning to possible topological terms, we consider differential forms we can construct using $g$. Given $-i \operatorname{Tr}\left(\sigma_{3} g^{-1} d g\right)$, we can construct the two-form

$$
\omega=d\left(-i \operatorname{Tr}\left(\sigma_{3} g^{-1} d g\right)\right)=i \operatorname{Tr}\left(\sigma_{3}\left(g^{-1} d g\right)^{2}\right) .
$$

The spatial components of this correspond to the vorticity with the identification (30). Further, we have the three-form $-i \operatorname{Tr}\left(\sigma_{3} g^{-1} d g\right) \wedge \omega$, which is proportional to $\operatorname{Tr}\left(g^{-1} d g\right)^{3}$ for dimensional reasons. The integral of $\operatorname{Tr}\left(g^{-1} d g\right)^{3}$ is the helicity of the fluid and is known to commute with all observables if we use the standard commutation rules for a fluid. Since $\omega \wedge \omega$ is zero (for dimensional reasons), some of the interesting topological terms we can construct using $g$ are as follows:

(1) $I_{1}=\int \omega \wedge B, B=$ two-form in $3+1$, one-form in $2+1$ dimensions;

(2) $I_{2}=\int \operatorname{Tr}\left(g^{-1} d g\right)^{3} \wedge C, C=$ one-form in $3+1$, zero-form in $2+1$ dimensions;

(3) $I_{3}=\int \operatorname{Tr}\left(\sigma_{3} g^{-1} d g\right) \wedge \Omega, \Omega=$ three-form in $3+1$, two-form inn $2+1$ dimensions.

The first one, namely $I_{1}$, is easy to dispose of. Since $\omega=d\left(-i \operatorname{Tr}\left(\sigma_{3} g^{-1} d g\right)\right)$, an integration by parts shows that $I_{1}$ is a surface term if $B$ is a closed form. Thus it will not affect the equations of motion or the canonical structure in the bulk. We will assume boundary conditions such that the surface term is zero. If $B$ is not a closed form, it reduces to $I_{3}$, with $\Omega=d B$.

Turning to $I_{2}$, notice that the variation of $\operatorname{Tr}\left(g^{-1} d g\right)^{3}$ is a total derivative and hence $I_{2}$ will not contribute to the equations of motion if $C$ is closed, i.e., $d C=0$. By considering the term with time derivatives of $g$ in $I_{2}$, we can see that its contribution to the canonical one-form is

$$
\Delta \mathcal{A}=-3 \int \operatorname{Tr}\left[g^{-1} \delta g d\left(g^{-1} d g\right)\right] \wedge C .
$$

This leads to $\delta(\Delta \mathcal{A})=-3 \int d\left[\left(g^{-1} \delta g\right)^{2} g^{-1} d g\right] \wedge C$, so that, if $C$ is closed, $I_{2}$ does not contribute to the canonical two-form either. (Again, we assume boundary conditions where the surface term does not contribute.) In other words $\Delta \mathcal{A}$ is a flat connection on the space of configurations $\{g(x)\}$. While it does not affect the Poisson brackets of observables, it does lead to a vacuum angle (via a term like $\theta I_{2}$ ), characterizing the state of the fluid in the quantum theory. 
If $C$ is not closed, we can have a nonzero $\delta(\Delta \mathcal{A})$. In this case, other than an external field, a natural choice for $C$ would be something like $\operatorname{Tr}\left(\sigma_{3} h^{-1} d h\right)$, where $h \in(S U(1,1)$ refers to another fluid. Thus $I_{2}$ will be a topological term coupling two fluids. However it is then of the form $I_{3}$ with $\Omega=\operatorname{Tr}\left(h^{-1} d h\right)^{3}$ if we further reverse the roles of the two fluids with an exchange $g \leftrightarrow h$.

The interesting cases to emerge from this analysis are thus (a) $I_{3}$ with $\Omega$ external, (b) $I_{2}$ with $C$ external, with $d C \neq 0$, (c) $I_{2}$ with $C \sim \operatorname{Tr}\left(\sigma_{3} h^{-1} d h\right)$, which is also the same as $I_{3}$ with $\Omega=\operatorname{Tr}\left(g^{-1} d g\right)^{3}$ with an exchange of $g$ and $h$. We will now analyze these cases in some detail below.

\section{THE TERM $I_{3}$ IN $3+1$ AND $2+1$ DIMENSIONS}

We now consider a fluid where, in addition to the usual terms, we add a term proportional to $I_{3}$ in the action. Thus the action is taken to be of the form

$S=-i \int \rho \operatorname{Tr}\left(\sigma_{3} g^{-1} \partial_{0} g\right)+i k \int \operatorname{Tr}\left(\sigma_{3} g^{-1} d g\right) \wedge \Omega-\int d t H$.

Here $k$ is a constant and $\Omega$ is a three-form for fluids in $3+1$ dimensions, and a two-form in $2+1$ dimensions. If $\Omega$ has a time component, then $g^{-1} d g$ in the extra term will be a spatial derivative and will not contribute to the canonical structure. So, for our purpose, we will assume that $\Omega$ is a three-form/two-form on the spatial manifold. Any time components can be added to our analysis without affecting the canonical structure which is the focus of our work. For brevity we write

$$
\bar{\rho}=k\left\{\begin{array}{ll}
\frac{1}{3 !} \epsilon^{i j k} \Omega_{i j k} & (3+1 \text { dimensions }) \\
\frac{1}{2 !} \epsilon^{i j} \Omega_{i j} & (2+1 \text { dimensions })
\end{array} .\right.
$$

[We are taking the dual of $\Omega$ to get $\bar{\rho}$, so that there should be a factor of $\left(\operatorname{det} g_{\mu \nu}\right)^{-1 / 2}$ in curved space, where $g_{\mu \nu}$ is the metric tensor.] The action (39) can be written as

$$
S=-i \int(\rho-\bar{\rho}) \operatorname{Tr}\left(\sigma_{3} g^{-1} \partial_{0} g\right)-\int d t H .
$$

The canonical one-form and two-form are given by

$$
\begin{aligned}
\mathcal{A} & =-i \int(\rho-\bar{\rho}) \operatorname{Tr}\left(\sigma_{3} g^{-1} \delta g\right), \\
\delta \mathcal{A} & =-i \int \delta \rho \operatorname{Tr}\left(\sigma_{3} g^{-1} \delta g\right)+i \int(\rho-\bar{\rho}) \operatorname{Tr}\left(\sigma_{3} g^{-1} \delta g g^{-1} \delta g\right) .
\end{aligned}
$$

It is now straightforward to work out a number of Poisson brackets. Consider a vector field $L(\theta)$ which corresponds to left translations on $g$ given by $V_{L}(\theta) g=-i \theta^{a} t_{a} g$. Contracting this vector field with $\delta \mathcal{A}$, we find

$$
\left.V_{L}(\theta)\right\rfloor \delta \mathcal{A}=\delta\left[\int(\rho-\bar{\rho}) \operatorname{Tr}\left(\sigma_{3} g^{-1} \theta^{a} t_{a} g\right)\right]
$$

which corresponds to the Poisson bracket relation

$$
\begin{aligned}
& {[L(\theta), g(x)]=i \theta^{a}(x) t_{a} g(x),} \\
& L(\theta)=-\int(\rho-\bar{\rho}) \operatorname{Tr}\left(\sigma_{3} g^{-1} t_{a} g\right) \theta^{a} .
\end{aligned}
$$

In a similar way, it is easy to obtain the relation

$$
[\rho(f), g(x)]=-i g(x) t_{3} f(x) .
$$

We now turn to spatial diffeomorphisms given by a vector field $V_{\xi}$ defined by

$$
V_{\xi} g=\xi^{i} \partial_{i} g, \quad V_{\xi} \rho=\nabla \cdot[\xi(\rho-\bar{\rho})] .
$$

(We consider $\bar{\rho}$ to be spatially constant for this.) The contraction of this vector field with $\delta \mathcal{A}$ gives

$$
\begin{aligned}
\left.V_{\xi}\right\rfloor \delta \mathcal{A} & =-\delta \mathcal{T}(\xi), \\
\mathcal{T}(\xi) & =-i \int(\rho-\bar{\rho}) \operatorname{Tr}\left(\sigma_{3} g^{-1} \xi \cdot \partial g\right) \\
& =\int(\rho-\bar{\rho}) \xi^{i} v_{i},
\end{aligned}
$$

where $v_{i}=-i \operatorname{Tr}\left(\sigma_{3} g^{-1} \partial_{i} g\right)$ is the fluid velocity as in (26). $\mathcal{T}(\xi)$ is thus the canonical generator of diffeomorphisms and it obeys the Poisson bracket algebra

$\left[\mathcal{T}(\xi), \mathcal{T}\left(\xi^{\prime}\right)\right]=\mathcal{T}\left(\left[\xi, \xi^{\prime}\right]\right), \quad\left[\xi, \xi^{\prime}\right]^{i}=\xi \cdot \partial \xi^{\prime i}-\xi^{\prime} \cdot \partial \xi^{i}$.

This algebra is as expected for diffeomorphisms. However, if we define the energy-momentum tensor by varying the action with respect to the metric, it has no contribution from the topological term, and we find

$$
\begin{aligned}
T(\xi) & =\int \xi^{i} T_{i 0}=\int \rho \xi^{i} v_{i}=-i \int \rho \operatorname{Tr}\left(\sigma_{3} g^{-1} \xi \cdot \partial g\right) \\
& =\mathcal{T}(\xi)+\int \bar{\rho} \xi^{i} v_{i} .
\end{aligned}
$$

From the Poisson bracket relations given above, we can easily verify that

$$
\begin{aligned}
\int \xi \cdot v\left[(\rho-\bar{\rho}), \int \xi^{\prime i} v_{i}\right] & =-\int \xi^{\prime} \cdot \partial(\xi \cdot v), \\
{\left[v_{i}(x), v_{j}(y)\right] } & =-\frac{1}{\rho-\bar{\rho}}\left(\partial_{i} v_{j}-\partial_{j} v_{i}\right) \delta(x-y) .
\end{aligned}
$$


Using these relations, the Poisson bracket algebra for $T(\xi)$ can be worked out as

$$
\begin{aligned}
& {\left[T(\xi), T\left(\xi^{\prime}\right)\right]} \\
& \quad=T\left(\left[\xi, \xi^{\prime}\right]\right)-\int\left(\frac{\rho \bar{\rho}}{\rho-\bar{\rho}}\right) \xi^{i} \xi^{\prime j}\left(\partial_{i} v_{j}-\partial_{j} v_{i}\right) .
\end{aligned}
$$

We see that the algebra for $T(\xi)$ has an extension term involving the density and the vorticity $\omega_{i j}=\partial_{i} v_{j}-\partial_{j} v_{i}$. This extension is absent for $\mathcal{T}(\xi)$ which is obtained by adding the integral of $-\bar{\rho} \xi \cdot v$ to $T(\xi)$. Since this extra term is well defined on the space of field configurations, the extension in the algebra (51) is not a true anomaly. To put this another way, it is cohomologically trivial, since it can be removed by a redefinition of the generators. So far this is in keeping with the absence of gravitational anomalies in $3+1$ and $2+1$ dimensions.

However, we can consider a reduction of the algebra (51) to the case of an incompressible fluid where we set $\rho-\bar{\rho}$ to some constant $\rho_{0}$; i.e., we impose a constraint

$$
\rho-\bar{\rho}-\rho_{0} \approx 0 .
$$

In the canonical reduction, we need a conjugate constraint, which may be taken as $\theta \approx 0$, where $\theta$ is the Clebsch variable in (26) and (29). The phase space is reduced to the set of all maps from space into $S U(1,1) / U(1)$. The fluid velocity $-i \operatorname{Tr}\left(\sigma_{3} g^{1} \partial_{i} g\right)$ is not invariant under a shift of $\theta$ and hence does not descend to the reduced space. The addition of the integral of $-\bar{\rho} \xi \cdot v$ to $T(\xi)$ is not defined on the reduced space and so the extension in (51) becomes a true anomaly. This is very similar to what we found for the sigma model in Sec. II.

Strictly speaking, we should also reduce the Poisson bracket algebra to an algebra for Dirac brackets to see if there is any change in the extension. But notice that the Poisson bracket of $T(\xi)$ with the constraint $\left(\rho-\bar{\rho}-\rho_{0}\right)$ vanishes on the constrained space since

$$
\begin{aligned}
& {\left[T(\xi), \int f\left(\rho-\bar{\rho}-\rho_{0}\right)\right]} \\
& \quad=\int \rho \xi \cdot \partial f \\
& \quad=\int\left(\rho-\bar{\rho}-\rho_{0}\right) \xi \cdot \partial f-\int f\left(\bar{\rho}+\rho_{0}\right) \nabla \cdot \xi \\
& \quad \approx 0
\end{aligned}
$$

for divergence-free vector fields $\xi^{i}$. (When we consider incompressible fluids only diffeomorphisms by divergencefree vector fields are meaningful.) As a result of this relation, the Dirac bracket $\left[T(\xi), T\left(\xi^{\prime}\right)\right]_{*}$ has the same right-hand side as in (51).

\section{PHYSICAL EXAMPLES OF THE $I_{3}$ TERM}

In this section we will consider specific physically interesting cases for which the $I_{3}$ topological term can be used.

\section{A. The vortex fluid in $\mathbf{2}+\mathbf{1}$ dimensions}

Vortices in a fluid are known to have many interesting properties. A particularly noteworthy feature is that their position variables in two spatial dimensions (or the two transverse position variables in three dimensions) form a canonically conjugate set, a result going back to Kirchhoff [12]. Recently Wiegmann, and Wiegmann and Abanov studied the hydrodynamic description of a large number of vortices in $2+1$ dimensions, in the physical contexts of superfluids and the quantum Hall effect [1]. The dynamics of this vortex fluid can be extracted from the Kirchhoff description of individual vortices and the dynamics of the underlying fluid. The number density to be used for the vortex fluid is related via a constitutive-type equation to the vorticity of the underlying fluid. A background overall rotation is introduced to cancel the vorticity to a large extent so that a separation of scales, with the underlying fluid having fast dynamics and the vortex fluid as the system of slow dynamics, is possible. Here we will not discuss more details of how the vortex fluid dynamics is extracted, for that the reader is referred to the papers cited, but we will give the key results relevant to comparison with our work.

In $2+1$ dimensions, it is natural to use complex coordinates $z, \bar{z}=x_{1} \pm i x_{2}$, with derivatives $\partial, \bar{\partial}=\frac{1}{2}\left(\partial_{1} \mp i \partial_{2}\right)$. The holomorphic component of the fluid velocity is taken as $u=u_{1}-i u_{2}$. The algebra of various observables can be summarized by the commutation rules

$$
\begin{aligned}
{[u(x), \rho(y)] } & =-i \partial \delta(x-y), \\
{\left[u(x), u^{\dagger}(y)\right] } & =-\frac{2 \pi}{\nu} \delta(x-y),
\end{aligned}
$$

where $\nu$ is a constant related to the strength $\Gamma$ of the individual vortices via $\nu=1 / \Gamma$. (It may also be interpreted as the filling fraction in the context of the Hall effect.) Diffeomorphisms are generated by the operators

$$
P=\rho u+\frac{1}{2 \nu} \partial \rho, \quad P^{\dagger}=u^{\dagger} \rho-\frac{i}{2 \nu} \bar{\partial} \rho .
$$

Defining $P(w)=\int w P, P^{\dagger}(w)=\int \bar{w} P^{\dagger}$, with complex test functions $w, \bar{w}$, the commutation rule for $P, P^{\dagger}$ can be obtained from (54) as 


$$
\begin{aligned}
{\left[P(w), P^{\dagger}\left(w^{\prime}\right)\right]=} & i \int\left(\bar{w}^{\prime} \bar{\partial} w P-w \partial \bar{w}^{\prime} P^{\dagger}\right) \\
& +\int \bar{w}^{\prime} w\left(i \rho\left(\bar{\partial} u-\partial u^{\dagger}\right)-\frac{2 \pi}{\nu} \rho^{2}\right) \\
& +\frac{1}{2 \nu} \int\left(\bar{w}^{\prime} \bar{\partial} w \partial \rho+w \partial \bar{w}^{\prime} \bar{\partial} \rho\right. \\
& \left.+\rho\left(w \bar{\partial} \partial \bar{w}^{\prime}+\bar{w}^{\prime} \bar{\partial} \partial w\right)\right) .
\end{aligned}
$$

Further simplification of the right-hand side can be done using the constitutive relation from [1]. It is given by

$$
i\left(\bar{\partial} u-\partial u^{\dagger}\right) \equiv(\nabla \times u)=\frac{2 \pi}{\nu}(\rho-\bar{\rho})
$$

where $\bar{\rho}=\nu \Omega / \pi$, with $\Omega$ being the angular velocity of the overall rotation. The commutation rule (56) now becomes

$$
\begin{aligned}
{\left[P(w), P^{\dagger}\left(w^{\prime}\right)\right]=} & i \int\left(\bar{w}^{\prime} \bar{\partial} w P-w \partial \bar{w}^{\prime} P^{\dagger}\right) \\
& -\frac{2 \pi}{\nu} \int \bar{w}^{\prime} w \rho \bar{\rho}-\frac{1}{\nu} \int \bar{\partial} w \partial \bar{w}^{\prime} \rho .
\end{aligned}
$$

In comparing this result with what was obtained in (51), we first note that, in two spatial dimensions, we have the freedom of adding to $T(\xi)$ a term proportional to the density, so that we can consider the more general quantity

$$
\tilde{T}(\xi)=T(\xi)+b \int(\nabla \times \xi) \rho
$$

where $b$ is a constant. This is essentially the same as the addition of $\partial \rho, \bar{\partial} \rho$ terms in defining $P, P^{\dagger}$ as in (55). The Poisson algebra for $\tilde{T}(\xi)$ can be easily worked out from (51) and the other relations given in the last section as

$$
\begin{aligned}
{\left[\tilde{T}(\xi), \tilde{T}\left(\xi^{\prime}\right)\right]=} & \tilde{T}\left(\left[\xi, \xi^{\prime}\right]\right)-\int\left(\frac{\rho \bar{\rho}}{\rho-\bar{\rho}}\right) \omega \epsilon^{i j} \xi_{i} \xi_{j}^{\prime} \\
& -b \int \rho \epsilon^{i j}\left(\partial_{k} \xi_{i} \partial_{k} \xi_{j}^{\prime}+\partial_{i} \xi_{k} \partial_{j} \xi_{k}^{\prime}\right)
\end{aligned}
$$

where $\omega=\partial_{1} v_{2}-\partial_{2} v_{1}$ is the two-dimensional vorticity. We now introduce test functions $w, \bar{w}$ via $w, \bar{w}=\xi_{1} \pm i \xi_{2}$ so that

$$
\begin{aligned}
\tilde{T}(\xi) & =-\int\left(w P+\bar{w} P^{\dagger}\right), \\
P & =-\frac{1}{2}\left(\tilde{T}_{01}-i \tilde{T}_{02}\right), \quad P^{\dagger}=-\frac{1}{2}\left(\tilde{T}_{01}+i \tilde{T}_{02}\right) .
\end{aligned}
$$

It is a bit tedious but straightforward to write (60) in terms of the complex test functions. We find

$$
\begin{aligned}
{\left[P(w), P^{\dagger}\left(w^{\prime}\right)\right]=} & i \int\left(\bar{w}^{\prime} \bar{\partial} w P-w \partial \bar{w}^{\prime} P^{\dagger}\right) \\
& +\frac{1}{2} \int\left(\frac{\rho \bar{\rho}}{\rho-\bar{\rho}}\right) \omega \bar{w}^{\prime} w+2 b \int \rho \bar{\partial} w \partial \bar{w}^{\prime} .
\end{aligned}
$$

We have also converted our Poisson bracket relations to commutators for operators by the appropriate multiplication by $i$, for ease of comparison. For us, the velocity of the fluid obeys the commutation rule $\left[\rho v_{i}(x), \rho(y)\right]=$ $i \rho \partial_{i} \delta(x-y)$. In comparing this with (54), we see that we must make the identification $u \equiv u_{1}-i u_{2}=$ $-\frac{1}{2}\left(v_{1}-i v_{2}\right)$, which leads to $\nabla \times u=-\frac{1}{2} \omega$. The constitutive relation (57) in our notation is thus

$$
\omega=-\frac{4 \pi}{\nu}(\rho-\bar{\rho}) .
$$

When this relation is used in (62), we see that we have exact agreement with (58), with $b=-\frac{1}{2 \nu}$.

What we have shown in this subsection may be summarized as follows. Consider the action

$$
\begin{aligned}
S= & -i \int \rho \operatorname{Tr}\left(\sigma_{3} g^{-1} \partial_{0} g\right)+i k \int \operatorname{Tr}\left(\sigma_{3} g^{-1} d g\right) \wedge \Omega-\int d t H \\
& +\int A_{0} d t\left[i \operatorname{Tr}\left[\sigma_{3}\left(g^{-1} d g\right)^{2}\right]+\frac{4 \pi}{\nu}(\rho-\bar{\rho})\right]
\end{aligned}
$$

where $k=1 /(\pi \Gamma)=\nu / \pi$. The two-form $\Omega$ (or its component $\Omega_{12}$ ) is to be interpreted as the angular velocity of overall rotation, and $\bar{\rho}=k \Omega_{12}$ as in (40). The last term in (63) has a Lagrange multiplier field $A_{0}$, which enforces the constitutive relation (63). Our result is that this action (64) describes the effective fluid dynamics of a vortex fluid in $2+1$ dimensions; it leads to the commutation rules (58) or (62). What we have obtained is thus an action formulation for the extended algebra (58), in much the same way as the topological term of the Wess-Zumino-Witten (WZW) model leads to the central extension of the Kac-Moody algebra [13].

We close this section with a comment clarifying the comparison with [1]. The commutation rules (54) are exactly those given in [1], so the algebra (58) follows by direct computation. However, the extension term as displayed in [1] is slightly different from the term in (58), involving the Laplace operator rather than holomorphic and antiholomorphic derivatives on $\bar{w}^{\prime}$ and $w$. We expect that the reason for this is the following. In [1], the quantization of the fluid is considered where the ground state obeys the condition $P|0\rangle=\langle 0| P^{\dagger}=0$. This is like a holomorphicity condition and, effectively, should be equivalent to a holomorphicity condition on the test function $w$. The algebra given in [1], written out with such test functions, then reduces to (58). 


\section{B. The vortex fluid in $3+1$ dimensions}

It is also interesting to consider a vortex fluid in $3+1$ dimensions with a constitutive relation similar to (63). The second extension to the algebra (58) arising from the addition of $\int \rho(\nabla \times \xi)$ to $T(\xi)$ is irrelevant for this case; it is trivial from the point of view of the cohomology of the algebra anyway, so we will focus on (51). We write the vorticity as

$$
\omega_{i j}=\partial_{i} v_{j}-\partial_{j} v_{i}=\epsilon_{i j k} N^{k} \omega
$$

where $N^{k}$ is a unit vector giving the orientation of the vorticity at a given point and $\omega$ is its magnitude. Unlike in two dimensions, we now have vortex lines, so $N^{k}$ gives the local orientation of a set of vortex lines coarse-grained over a small volume. As in the $(2+1)$-dimensional case, we expect $\omega$ to be proportional to the number density of vortices. So we propose to use the same constitutive relation in three dimensions as well, namely,

$$
\omega=-\frac{4 \pi}{\nu}(\rho-\bar{\rho})
$$

where $\bar{\rho}$ is given in terms of the three-form $\Omega$ as in (40). The algebra (51) takes the form

$$
\begin{aligned}
{\left[T(\xi), T\left(\xi^{\prime}\right)\right] } & =T\left(\left[\xi, \xi^{\prime}\right]\right)+\int \epsilon_{i j k} \xi^{i} \xi^{\prime j} c^{k} \\
c^{k} & =\frac{4 \pi}{\nu} \rho \bar{\rho} N^{k}
\end{aligned}
$$

We will now relate this to some recent work on the algebra of vector fields for an incompressible fluid [10]. If we consider the reduction of the algebra (67) to the incompressible case, with the vector fields $\xi^{i}, \xi^{\prime j}$ being divergence-free, $\rho \bar{\rho}$ can be taken to be a constant. The motion of the vortices is on a two-dimensional surface transverse to their vortex lines, i.e., transverse to the vector $N^{k}$. If we have a large dense collection of vortices, $N^{k}$ will be uniform in the transverse surface, just as it was in $2+1$ dimensions. As one follows along the vortex lines, $N^{k}$ can change orientation. It is useful to consider the case of $N^{k}$ being constant, independent of $\vec{x}$. This would be realizable at least in some subvolume of space. In this case, the extension term in (67) becomes

$$
\text { Extension }=c^{k} \int \epsilon_{i j k} \xi^{i} \xi^{j} .
$$

If space is taken to be a 3-torus as in [10], one can parametrize the divergence-free vector fields as

$$
\xi^{i}=\epsilon^{i a b} \alpha_{a} m_{b} e^{i \vec{m} \cdot \vec{x}}, \quad \xi^{\prime j}=\epsilon^{j r s} \beta_{r} n_{s} e^{i \vec{n} \cdot \vec{x}} .
$$

Here $m_{i}, n_{i}$ are vectors of integer components and $e^{i \vec{m} \cdot \vec{x}}$, $e^{i \vec{n} \cdot \vec{x}}$ provide a basis for functions on the torus. By taking the components of each of $\alpha_{i}, \beta_{i}$ and $(\vec{\alpha} \times \vec{\beta})_{i}$ to be linearly independent over the integers, one can get a dense set of test functions. With the test functions in (69), the extension term (68) becomes

$$
\text { Extension }=-(\vec{\alpha} \times \vec{\beta}) \cdot \vec{n} \vec{c} \cdot \vec{n} \text {. }
$$

This is in agreement with the central extension considered in [10]. So our conclusion is that the topological term $I_{3}$ can explain the central extension of [10] as a special case with the reduction conditions as explained above. The consideration of a constant $c^{k}$ was just for showing this connection. But using the action (39) we can go beyond considering constant $c^{k}$, with the more general algebra (67) being applicable to a vortex fluid in $3+1$ dimensions. The extension is no longer a central term in the general case.

\section{THE TERM $I_{2}$ IN $3+1$ DIMENSIONS}

We now turn to the second case of a topological term we listed at the end of Sec. III, namely, $I_{2}=\operatorname{Tr}\left(g^{-1} d g\right)^{3} \wedge C$ where $C$ will be taken as external, with $d C \neq 0$. Adding such a term with coefficient $-k / 3$, the action we are considering is

$$
S=-i \int \rho \operatorname{Tr}\left(\sigma_{3} g^{-1} \partial_{0} g\right)-\frac{k}{3} \int \operatorname{Tr}\left(g^{-1} d g\right)^{3} \wedge C-\int d t H .
$$

The terms with the time derivative of $g$ lead to the canonical one-form and two-form

$$
\begin{aligned}
\mathcal{A}= & -i \int \rho \operatorname{Tr}\left(\sigma_{3} g^{-1} \delta g\right)-k \int \operatorname{Tr}\left[g^{-1} \delta g\left(g^{-1} d g\right)^{2}\right] \wedge C \\
\delta \mathcal{A}= & -i \int\left[\delta \rho \operatorname{Tr}\left(\sigma_{3} g^{-1} \delta g\right)-\rho \operatorname{Tr}\left(\sigma_{3}\left(g^{-1} \delta g\right)^{2}\right]\right. \\
& +k \int \operatorname{Tr}\left[\left(g^{-1} \delta g\right)^{2} g^{-1} d g\right] \wedge C .
\end{aligned}
$$

In this case the identification of the Hamiltonian vector fields is not very easy; it is simpler to work out the inverse of the canonical two-form and form the Poisson brackets. Towards this, we write

$$
\begin{aligned}
& g^{-1} \delta g=-i t_{a} \mathcal{E}^{a}=-i t_{a} \mathcal{E}_{\alpha}^{a} \delta \varphi^{\alpha}, \\
& g^{-1} d g \equiv-i t_{a} I^{a} .
\end{aligned}
$$

The canonical two-form $\delta \mathcal{A}$ is then given as

$\delta \mathcal{A}=-\delta \rho \mathcal{E}^{3}+\frac{1}{2} \rho \epsilon_{a b 3} \mathcal{E}^{a} \wedge \mathcal{E}^{b}-\frac{1}{2} \epsilon_{a b c} b^{c} \mathcal{E}^{a} \wedge \mathcal{E}^{b}$

where 


$$
b^{c}=\frac{k}{2} B_{i} I_{i}^{c}, \quad B^{k}=\epsilon^{k l m} \partial_{l} C_{m} .
$$

Taking the inverse of $\delta \mathcal{A}$, the Poisson bracket of functions $A, B$ is given as

$$
\begin{aligned}
{[A, B]=} & \int\left[\frac{\delta A}{\delta \rho(x)} \mathcal{E}_{3}^{-1 \alpha} \frac{\delta B}{\delta \varphi^{\alpha}(x)}-\frac{b_{1}}{\rho-b_{3}} \frac{\delta A}{\delta \rho(x)} \mathcal{E}_{1}^{-1 \alpha} \frac{\delta B}{\delta \varphi^{\alpha}(x)}\right. \\
& -\frac{b_{2}}{\rho-b_{3}} \frac{\delta A}{\delta \rho(x)} \mathcal{E}_{2}^{-1 \alpha} \frac{\delta B}{\delta \varphi^{\alpha}(x)} \\
& \left.-\frac{1}{\rho-b_{3}} \frac{\delta A}{\delta \varphi^{\alpha}} \mathcal{E}_{1}^{-1 \alpha} \mathcal{E}_{2}^{-1 \beta} \frac{\delta B}{\delta \varphi^{\beta}}-(A \leftrightarrow B)\right]
\end{aligned}
$$

The computation of the Poisson brackets of various quantities of interest using this formula is tedious but straightforward. The bracket of $\rho$ and $g$ is given by

$[\rho(f), g(x)]=-i\left[\frac{\rho}{\rho-b_{3}} g t_{3}-\frac{1}{\rho-b_{3}} g b^{a} t_{a}\right] f(x)$.

As before, using the energy-momentum tensor obtained by varying (71) with respect to the metric, we have

$$
T(\xi)=-i \int \rho \operatorname{Tr}\left(\sigma_{3} g^{-1} \xi \cdot \partial g\right)=\int \rho \xi \cdot v
$$

For this we have the Poisson bracket algebra

$$
\begin{aligned}
{[\rho(f), T(\xi)]=} & -\int \rho \xi \cdot \nabla f \\
& -\int \frac{\rho}{\rho-b_{3}} \operatorname{Tr}\left(\left[\sigma_{3}, b^{a} t_{a}\right]\left(g^{-1} \xi \cdot \partial g\right)\right) f \\
{\left[T(\xi), T\left(\xi^{\prime}\right)\right]=} & T\left(\left[\xi, \xi^{\prime}\right]\right) \\
& +k \int \frac{\rho}{\rho-b_{3}} \operatorname{Tr}\left(\left[g^{-1} \xi \cdot \partial g, g^{-1} \xi^{\prime} \cdot \partial g\right] g^{-1} d g\right) \\
& \wedge d C .
\end{aligned}
$$

Recall that the helicity of the fluid is given by

$$
\mathcal{C}=\frac{1}{8 \pi} \int v \cdot \omega=\frac{1}{12 \pi} \int \operatorname{Tr}\left(g^{-1} d g\right)^{3} \equiv \int \sigma .
$$

In terms of the density $\sigma$ for helicity as defined above, the bracket relation for $T(\xi)$ can be written as

$$
\left[T(\xi), T\left(\xi^{\prime}\right)\right]=T\left(\left[\xi, \xi^{\prime}\right]\right)+4 \pi k \int\left(\frac{\rho \sigma}{\rho-b_{3}}\right)(\vec{\xi} \times \vec{\xi}) \cdot \vec{B}
$$

A natural question at this point would be whether there is a physical system for which the present case of $I_{2}$ is applicable. The example of vortex fluids discussed in the last section can be a guide in this direction. As known for a long time, helicity is related to knots and links for vortex lines. For example, consider the linking of a vortex line with another, the latter forming a circle which may be viewed as the boundary of some two-surface $\Sigma$. If vortices are approximated by thin lines, the integrand $\sigma$ for helicity has support at the point of intersection of the vortex line with the surface $\Sigma$. The support for $\sigma$ is pointlike localized in the thin vortex approximation. As time evolves, these points can move and for a fluid with a dense collection of such knots one can envisage constructing an effective hydrodynamics of knots or links. The action with the $I_{2}$ term added as in (71) is a good candidate for such an effective hydrodynamics. Again an overall rotation (interpreted as $d C$ ) may be needed to ensure a proper separation of scales. Although well motivated, admittedly, this connection is still speculative; it will need more work to tie down the specifics.

\section{DISCUSSION}

In this paper, we considered some topological terms which can be added to the standard actions for sigma models and for fluid dynamics. The example of the sigma model with $\mathbb{C P}^{2}$ as the target space shows how the additional term can lead to a conflict between diffeomorphisms for the target and base spaces. For the case of fluid dynamics, it is worth emphasizing that we are not introducing any additional variables or degrees of freedom. We use the standard action in terms of the Clebsch variables with the topological terms added. In $2+1$ dimensions, we showed how one such topological term leads to the effective hydrodynamics of a vortex fluid as derived in [1]. This provides an action-based derivation of the extended diffeomorphism algebra in much the same way as the WZW model gives an action-based derivation of the central extension of the Kac-Moody algebra. A similar analysis was made in $3+1$ dimensions, presumably applicable to a fluid of vortex lines. A special case leads to the central extension found in [10]. We also discussed another topological term using the helicity of the fluid, which might apply to a fluid made of knots and links of vortex lines.

There are a couple of other relevant observations. For the example of the invariant $I_{2}$, we considered the one-form $C$ to be external, with $d C \neq 0$. As already mentioned, one could also consider $C$ to be of the form $\operatorname{Tr}\left(\sigma_{3} h^{-1} d h\right)$ where $h$ defines the Clebsch variables for another fluid. The algebra of observables for the second fluid will be similar to what was obtained for the $I_{3}$ invariant. However, for the combined system, there can be cross terms. These need further analysis. Our second observation is about the Hopf invariant. If one considers vortices of quantized charge or strength, the Clebsch variables are described by $S U(2)$ rather than $S U(1,1)$ with the vorticity as the pull-back to 
space of the volume form on $S^{2}=S U(2) / U(1)$. It is then natural to consider the Hopf invariant in $2+1$ dimensions, and in $3+1$ dimensions with an additional one-form $C$. A partial analysis of the $2+1$ case is given in [14], but more needs to be done for this case as well.

\section{ACKNOWLEDGMENTS}

I thank A. Abanov, G. Monteiro, and especially P. Wiegmann for many useful discussions. This research was supported in part by the U.S. National Science Foundation Grant No. PHY-1820721 and by PSC-CUNY awards.
[1] P. Wiegmann, arXiv:1211.5132; JETP 144, 617 (2013); Phys. Rev. B88, 241305(R) (2013); arXiv:1906.03788; P. Wiegmann and A. G. Abanov, Phys. Rev. Lett. 113, 034501 (2014).

[2] S-G. Jo, Phys. Lett. 163B, 353 (1985); B. Zumino, Nucl. Phys. B253, 477 (1985); L. Faddeev and S. L. Shatashvili, Phys. Lett. 167B, 225 (1986); T. Nishikawa and I. Tsutsui, Nucl. Phys. B308, 544 (1988); S. Ghosh and R. Banerjee, Phys. Lett. B 220, 581 (1989); Z. Phys. C 41, 121 (1988); G. V. Dunne and C. A. Trugenberger, Phys. Lett. B 248, 305 (1990); C. Schwiebert, Z. Phys. C 51, 93 (1991).

[3] L. Alvarez-Gaume and E. Witten, Nucl. Phys. B234, 269 (1984); see also R. Bertlmann, Anomalies in Quantum Field Theory (Oxford University Press, Oxford, 1996); S. Treiman, R. Jackiw, B. Zumino, and E. Witten, Current Algebra and Anomalies (World Scientific, Singapore, 1985).

[4] Fluid description of Hall states is an old topic with many articles devoted to it. For general background, see R. E. Prange and S. M. Girvin, The Quantum Hall Effect, 2nd ed. (Springer-Verlag, Berlin, 2012); Z. F. Ezawa, Quantum Hall Effects (World Scientific, Singapore, 2008); Some other relevant references include J. E. Avron, R. Seiler, and P. G. Zograf, Phys. Rev. Lett. 75, 697 (1995); N. Read, Phys. Rev. B 79, 045308 (2009); N. Read and E. H. Rezayi, Phys. Rev. B 84, 085316 (2011); F. D. M. Haldane, Phys. Rev. Lett. 51, 605 (1983); A. G. Abanov and A. Gromov, Phys. Rev. B 90, 014435 (2014); A. Gromov and A. Abanov, Phys. Rev. Lett. 113, 266802 (2014); A. Gromov, G. Cho, Y. You, A. G. Abanov, and E. Fradkin, Phys. Rev. Lett. 114, 016805 (2015); T. Can, M. Laskin, and P. Wiegmann, Phys. Rev. Lett. 113, 046803 (2014); Ann. Phys. (Amsterdam) 362, 752 (2015); S. Klevtsov and P. Wiegmann, Phys.
Rev. Lett. 115, 086801 (2015); B. Bradlyn and N. Read, Phys. Rev. B 91, 165306 (2015); S. Klevtsov, X. Ma, G. Marinescu, and P. Wiegmann, arXiv:1510.06720.

[5] For a review, see M. Rangamani, Classical Quantum Gravity 26, 224003 (2009) and references therein.

[6] For a review of fluid dynamics from a modern point of view, see R. Jackiw, V. P. Nair, S-Y. Pi, and A. P. Polychronakos, J. Phys. A 37, R327 (2004); B. Bistrovic, R. Jackiw, H. Li, V. P. Nair, and S. Y. Pi, Phys. Rev. D 67, 025013 (2003).

[7] There is a large body of literature on the chiral magnetic effect. Some of the key papers are D. Kharzeev, Phys. Lett. B 633, 260 (2006); D. Kharzeev and A. Zhitnitsky, Nucl. Phys. A797, 67 (2007); D. E. Kharzeev, L. D. McLerran, and H. J. Warringa, Nucl. Phys. A803, 227 (2008); K. Fukushima, D. E. Kharzeev, and H. J. Warringa, Phys. Rev. D 78, 074033 (2008); D. E. Kharzeev, Ann. Phys. (Amsterdam) 325, 205 (2010).

[8] V. P. Nair, Rashmi Ray, and Shubho Roy, Phys. Rev. D 86, 025012 (2012); D. Capasso, V. P. Nair, and J. Tekel, Phys. Rev. D 88, 085025 (2013).

[9] G. M. Monteiro, A. G. Abanov, and V. P. Nair, Phys. Rev. D 91, 125033 (2015).

[10] S. G. Rajeev, arXiv:2005.12125.

[11] H. Bateman, Proc. R. Soc. A 125, 598 (1929); C. C. Lin, International School of Physics E. Fermi (XXI), edited by G. Careri (Academic Press, New York, 1963), p. 93.

[12] Kirchhoff's work is classic and is already discussed in Lamb's book from 1895; see H. Lamb, Hydrodynamics (Cambridge University Press, Cambridge, England, 1895), later edition reprinted in 1997.

[13] E. Witten, Commun. Math. Phys. 92, 455 (1984); S. P. Novikov, Usp. Mat. Nauk 37, 3 (1982).

[14] V. P. Nair and R. Ray, Nucl. Phys. B676, 659 (2004). 